Original article

\title{
Understanding the three As (Awareness, Access, and Acceptability) dimensions of vaccine hesitancy in Odisha, India
}

\author{
Shantanu Sharma*, Faiyaz Akhtar, Rajesh Kumar Singh, Sunil Mehra \\ MAMTA Health Institute for Mother and Child, Delhi, India
}

\section{A R T I C L E I N F O}

\section{Keywords:}

Immunization

Measles

Timeliness

Vaccine hesitancy

Vaccine acceptance

\begin{abstract}
A B S T R A C T
Background: A considerable proportion of the population remain unvaccinated due to multiple reasons including vaccine hesitancy. To study the factors responsible for the incomplete immunization of under-5 children from the perspectives of the communities, a vaccine hesitancy survey was done in two districts of Odisha. In addition, the survey aimed to elucidate the reasons for differential vaccine hesitancy.

Methods: This community-based cross-sectional study was conducted in the rural areas of Balangir and Nuapada districts in Odisha. We adapted the vaccine hesitancy survey questionnaire (core closed questions) developed by SAGE working group. We used this questionnaire in a sample of 260 households, selected from 30 clusters. The questions were categorized into three main dimensions, namely, awareness about vaccine efficacy, accessibility to obtain vaccine, and acceptability of the vaccines at the individual and community level. We used bivariate and multivariate regression analysis to determine the predictors for refusal or hesitancy of the vaccine.

Results: Long distances and time are the two prime factors of accessibility linked with the refusal or hesitancy of the vaccine. We found that there is a high level of acceptance of vaccines at the level of parents and more than three-fourths took their children for vaccination despite hearing negative information about them. Pentavalent and measles were more commonly refused or hesitated vaccines by the parents compared to BCG or polio.

Conclusion: In the current scenario of increasing immunization coverage in India, vaccine hesitancy acts as a missing link in action for the policymakers to achieve cent percent target.
\end{abstract}

\section{Introduction}

Immunization has been one of the most successful and cost-effective public health interventions. ${ }^{1,2}$ In the last decade, there has been a significant improvement in the rate of immunization in Low Middle-Income countries. Still, 23.5 million children remain unimmunized, and 1.7 million children die from vaccine-preventable diseases every year globally. ${ }^{3}$ India alone contributes to $36.5 \%$ of the total number of unimmunized children globally. India has an annual cohort of 26.7 million infants. More than one-third of children failed to receive all basic vaccines in the first year of life in $2016 .{ }^{4}$ The government of India started a program, named as Intensified Mission Indradhanush in 2017 with a rigorous implementation strategy to target underserved, vulnerable, resistant, and inaccessible populations. ${ }^{5}$

Amidst the tremendous progress achieved in immunization, there are increasing public concerns related to vaccine safety or efficacy. Public decision-making related to the acceptance of vaccine is driven by a mix of scientific, psychological, sociocultural, and political reasons. ${ }^{2}$ Vaccine hesitancy defined as a delay in acceptance or refusal of vaccines despite the availability of services, is context specific and varies across geographies and vaccine types. ${ }^{6}$ There are several forms of vaccine hesitancy. At its worsts, parents may refuse to vaccinate their children with all recommended vaccines under the national immunization program. However, this is relatively rare, and refusal or delay of one or more vaccine is more common. Parental concerns about the immediate side effects of vaccines are largely responsible for vaccine delay or refusal. ${ }^{7,8}$

Situated in East of India, Odisha is a state with three-fourth of the population belonging to scheduled caste, or tribe or other backward classes. With infant mortality rate at 40 deaths per 1000 live births, Odisha is one of the lowest performing states in the health index composed by the National Institute of Transforming India. ${ }^{9}$ However, the state has achieved a considerable increase in the immunization coverage of under-5 children since the last decade. Multiple reasons for such an encouraging shift has been stated including improved supportive supervision of health workers, logistics management and self-belief of the communities in vaccines, etc. ${ }^{10}$ In this growing trend of improved vaccination coverage in Odisha, still, a large chunk of children (21\%)

\footnotetext{
${ }^{*}$ Corresponding author. B-5, Greater Kailash Enclave-2, Delhi, 110048, India.

E-mail address: shantanusharma@mamtahimc.org (S. Sharma).
} 
remained partially or completely unimmunized. ${ }^{4}$ To study the factors responsible for the incomplete immunization of under-5 children from the perspectives of the communities, a vaccine hesitancy survey was done in two districts of Odisha. In addition, the survey aimed to elucidate the reasons for differential vaccine hesitancy.

\section{Methods}

This community-based cross-sectional study was conducted in the rural areas of Balangir and Nuapada districts in Odisha. These were part of our implementation project, Project JAGRITI. Balangir, located in Odisha has a total area of $6575 \mathrm{~km}^{2}$ and has 14 blocks. The total population of the district is over 1.6 million. Nuapda has a total area of $3852 \mathrm{~km}^{2}$ and has 6 blocks. The total population of the district is over 0.6 million. ${ }^{11}$ A sample size of 211 households was calculated based on the World Health organization reference of cluster survey for immunization coverage. ${ }^{12}$ Two-stage cluster sampling was done to select study subjects. Thirty such clusters in two districts (20 in Balangir and 10 in Nuapada) were identified using area maps and with the support of local field teams. The first household in each cluster was selected randomly using the existing household list of families with under-5 children. Rest of the households consecutive to the first one were selected for the interview. Seven households from each cluster in Balangir and Nauapada were selected. An additional 49 households were approached to adjust for the non-response rate of $20 \%$. However, none of the households refused for the survey and the data from all the 260 households were analyzed $(211+49)$. Parents (preferably mother) with children under-5 years of age who lived in the area for at least 12 months prior to the survey were included in the study. We asked information related to the youngest child in the family.

We adapted the vaccine hesitancy survey questionnaire (core closed questions) developed by SAGE working group. ${ }^{13}$ The questionnaire had eleven questions in total and assessed parental awareness about vaccine efficacy, if they ever refused or were hesitant to vaccinate their child, vaccine types refused or hesitated by the respondents and reasons for the same, barriers of vaccination, receipt of support from political or religious leaders, etc. In addition, we obtained information about the socio-demographic characteristics of the family. The questionnaire was translated from English to Odiya, the local language, by a professional translator and verified by the researchers for linguistic equivalence of terms and concepts. The study was granted ethical approval by the Institutional ethical committee. Verbal informed consent was obtained from the parents. All included respondents were assured of the confidentiality of the data and informed about their rights to withdraw any time during the study.

The questions were categorized into three main domains, namely, awareness about vaccine efficacy, accessibility as a barrier to obtaining vaccine and acceptability of the vaccines at the individual and community level. Frequencies were expressed as percentages. The refusal or hesitancy was merged into one column (keeping either of them as an outcome) as a dependent variable for regression analysis. Bivariate analysis was done to look for the relationship between each explanatory variable and the refusal or hesitancy of vaccine using Pearson's chisquare test. The strength of association between variables was expressed as odds ratio (OR) and the corresponding $95 \%$ confidence interval $(95 \% \mathrm{CI})$. To determine the predictors for refusal or hesitancy of the vaccine, all the variables of accessibility, awareness, and acceptability with significance $(\mathrm{p}<0.05)$ in bivariate analysis were considered as eligible for inclusion in the multivariable logistic regression model. In the model, non-significant factors $(p<0.05)$ were eliminated by the backward stepwise method. The Hosmer-Lemeshow test (goodness of fit test) was done to test the calibration of the logistic regression model. Radar chart was used to express the prevalence of various reasons for the refusal or hesitancy of three vaccines (Bacillus Calmette Guarin (BCG), Pentavalent, and Measles). IBM SPPS statistics for windows version 24.0 (IBM Corp., Armonk, N.Y., USA) was used for
Table 1

District-wise distribution of responses of the families to vaccine hesitancy.

$\begin{array}{lll}\text { S.No. Variables } & \begin{array}{l}\text { Balangir } \\ (\mathrm{n}=180) \mathrm{N} \\ (\%)\end{array} & \begin{array}{l}\text { Nuapada } \\ (\mathrm{n}=80) \mathrm{N} \\ (\%)\end{array} \\ \end{array}$

Awareness

1. Does vaccine protect against infectious diseases?

Yes

$\begin{array}{ll}175(98) & 80(100) \\ 5(2) & 0\end{array}$

2. Should parents vaccinate their children against all recommended vaccines? Yes

80 (100)

No

Access

3. Has distance, timing of clinic, time needed to get to clinic or wait at clinic and/or costs in getting to clinic prevented you from getting your child immunized?

Distance

Time

19 (10.5)

22 (12.3)

7 (8.75)

Lack of Information about the place and 9 (5)

time

Nothing prevented

$130(72.2)$

$71(88.75)$

4. What pressures in life that prevent you from getting your child immunized?

Lack of time

Lack of support from family members

No pressures

$23(12.8)$

$1(0.5)$

0

156 (86.7) $80(100)$

Acceptance at the individual level

5. Any reasons that your child should not be vaccinated?

No need if child is healthy

$6(3.3)$

Child may develop complications like

fever

No reasons

$2(1.1)$

0

6. What negative information did you hear about vaccines?

Child may die

Child may develop complications like

fever

Don't know what is given to child

No negative information heard

9 (5)

38 (21.1)

79 (98.75)

0

7 (8.75)

$4(2.2)$

0

129 (71.7) 73 (91.25)

7. Did you take your child for vaccination even after hearing negative information?

Yes

$\mathrm{N}=51$

$\mathrm{N}=7$

$38(74.5)$

6 (85.7)

No

13 (25.5)

1 (14.3)

8. Have you ever been reluctant or hesitant to vaccinate your child?

Yes

$26(14.4)$

10 (12.5)

No

154 (85.6)

$70(87.5)$

9. Have you ever refused any vaccine? Yes

$23(12.8) \quad 10(12.5)$

No

$157(87.2) \quad 70(87.5)$

Acceptance at the community level

10. What difficulties are there for some

ethnic groups to vaccinate their children?

They choose not to vaccinate

They do not feel welcome at the health service

The health services don't reach them

No difficulties

11. Do leaders in your community support

vaccines for infants and children?

A. Religious leaders

Yes

No

Don't know

$112(62.2) \quad 42(52.5)$

$40(22.2) \quad 13(16.3)$

$28(15.6) \quad 25(31.3)$

B. Political leaders

Yes

$116(64.4) \quad 46(57.5)$

No

$40(22.2)$

Don't know

$24(13.3)$ 
all statistical analysis.

\section{Results}

A total of 180 (69.2\%) households from Balangir and 80 (30.8\%) households from Nuapada were taken in the survey. None of the eligible parents withheld consent to participate in the survey. Nearly $95 \%$ of the children were delivered in the hospital. Median (IQR) age of the children whose parents were surveyed was 17 (23) months. Out of the total children, $45 \%$ were boys and $55 \%$ girls. Nearly, three-fourth of the families belonged to backward classes (Scheduled caste or tribe), and the rest were from non-backward classes. Of the two-sixty households surveyed, 85\% had monthly income less than Indian Rupees 5000 (75 USD). Fifty-seven percent of respondents told that the main source of income is agriculture.

Table 1 describes the distribution of variables of awareness, accessibility, and acceptability of the vaccines by the households across two districts. All the households (100\%) in Nuapada knew that vaccines protect against infectious diseases and parents should vaccinate their children against all recommended vaccines and only $2 \%$ were not aware of them in Balangir. Around 11\% of parents from Balangir and 9\% from Nuapada highlighted long distance as an important barrier to vaccine uptake. They cited that due to lack of vaccine clinic in their village, they had to travel to another village, which is quite far. Parents informed that they had to wait for long hours at times and it had happened that they returned without vaccination. Nearly, 28\% parents in Balangir and $9 \%$ in Nuapada had heard negative information about the vaccines, and despite hearing negative information, more than three-fourth parents took their children to vaccination in both districts (Table 1).

In the bivariate analysis, factors related to awareness (can vaccine protect against infectious diseases), accessibility (distance or time prevented you from vaccination), acceptance at the individual level
Table 3

Prevalence of hesitancy or refusal across different vaccines (differential vaccine hesitancy).

\begin{tabular}{lll}
\hline Vaccines & Hesitate $(\mathrm{n}=36) \mathrm{N}(\%)$ & Refuse $(\mathrm{n}=33) \mathrm{N}(\%)$ \\
\hline BCG $^{\mathrm{a}}$ & $6(16.7)$ & $5(15)$ \\
Polio & $5(13.8)$ & $5(15)$ \\
Pentavalent & $15(41.7)$ & $13(39.4)$ \\
Measles & $10(27.8)$ & $12(36.3)$ \\
\hline
\end{tabular}

a BCG: Bacillus Calmette Guarin.

(any reasons that child should not be vaccinated, heard negative information) and acceptance at the community level (support from religious or political leaders) were significantly associated with refusal or hesitancy of vaccine (Table 2). Pentavalent and measles were more commonly refused or hesitated vaccines by the parents compared to BCG or polio (Table 3). Bad experiences by the parents with the vaccines and hearing negative information about vaccines or its reactions from others were commonly cited as reasons for refusal or hesitancy of pentavalent or measles vaccine. Some had even stated fear of needles as a reason to refuse or delay vaccine (Fig. 1).

\section{Discussion}

This study was instrumental in understanding the underlying factors of vaccine hesitancy in the two districts of Odisha. The study helped us channelize our interventions in the two districts to increase the immunization coverage. Most of the people knew that vaccines protect against infectious diseases and recommended vaccination for their children in our study. Similar studies from Chennai and West Bengal have stated about the increased awareness and positive beliefs of parents in vaccines. ${ }^{14,15}$ The major reasons for increased awareness about vaccines among parents could be widespread use of social media, mass

Table 2

Logistic regression (Bivariate and Multivariate) analysis showing associations between awareness, accessibility, and acceptability factors and refusal or hesitancy of the vaccines.

\begin{tabular}{|c|c|c|c|c|c|}
\hline \multirow[t]{2}{*}{ S.No. } & \multirow{2}{*}{$\begin{array}{l}\text { Variables } \\
\text { Independent variables }\end{array}$} & \multicolumn{2}{|l|}{ Unadjusted model } & \multicolumn{2}{|l|}{ Adjusted model } \\
\hline & & Odds ratio $(95 \% \mathrm{CI})$ & $\mathrm{P} *$ value & Odds ratio $(95 \% \mathrm{CI})$ & $\mathrm{P}^{*}$ value \\
\hline 1. & $\begin{array}{l}\text { Does vaccine protect against infectious diseases? } \\
\text { Yes } \\
\text { No }\end{array}$ & $0.38 \mathrm{e}-9$ & $<0.001$ & $2.3 e-9$ & $<0.001$ \\
\hline 2. & $\begin{array}{l}\text { Should parents vaccinate their children against all recommended vaccines? } \\
\text { Yes } \\
\mathrm{No}^{\text {厂 }}\end{array}$ & $0.16(0.05-0.5)$ & 0.002 & $1.6(0.12-20)$ & 0.7 \\
\hline 3. & $\begin{array}{l}\text { Has distance or timing prevented you from getting your child immunized? } \\
\text { Yes } \\
\mathrm{No}^{\text {厂 }}\end{array}$ & $6.8(3.2-14.5)$ & $<0.001$ & $5.7(2.2-14.9)$ & $<0.001$ \\
\hline 4. & $\begin{array}{l}\text { Did pressures in life prevent you from getting your child immunized? } \\
\text { Yes } \\
\text { No }\end{array}$ & $1.7(0.6-4.9)$ & 0.3 & - & - \\
\hline 5. & $\begin{array}{l}\text { Any reasons that child should not be vaccinated? } \\
\text { Yes } \\
\text { No }^{\text {q }}\end{array}$ & $26.8(5.3-135.0)$ & $<0.001$ & $14(1.3-151.0)$ & 0.03 \\
\hline 6. & $\begin{array}{l}\text { Any difficulties for ethnic groups to vaccinate their children? } \\
\text { Yes } \\
\mathrm{No}^{\text {厂 }}\end{array}$ & $3.3(1.5-7.4)$ & 0.003 & $1.1(0.3-3.5)$ & 0.8 \\
\hline 7. & $\begin{array}{l}\text { Did you hear negative information about vaccines? } \\
\text { Yes } \\
\mathrm{No}^{\top}\end{array}$ & $3.9(1.9-8.3)$ & $<0.001$ & $2.7(1.06-7.2)$ & 0.03 \\
\hline 8. & $\begin{array}{l}\text { Did religious leaders in your community support vaccines for infants and children? } \\
\text { Yes } \\
\text { No }^{\top}\end{array}$ & $0.25(0.1-0.5)$ & $<0.001$ & $4.7(0.5-4.3)$ & 0.17 \\
\hline 9. & $\begin{array}{l}\text { Did political leaders in your community support vaccines for infants and children? } \\
\text { Yes } \\
\text { No }\end{array}$ & $0.18(0.08-0.4)$ & $<0.001$ & $0.06(0.006-0.55)$ & 0.01 \\
\hline
\end{tabular}

*p value $<0.05$ is considered statistically significant.

†Reference category; CI: Confidence Interval. 


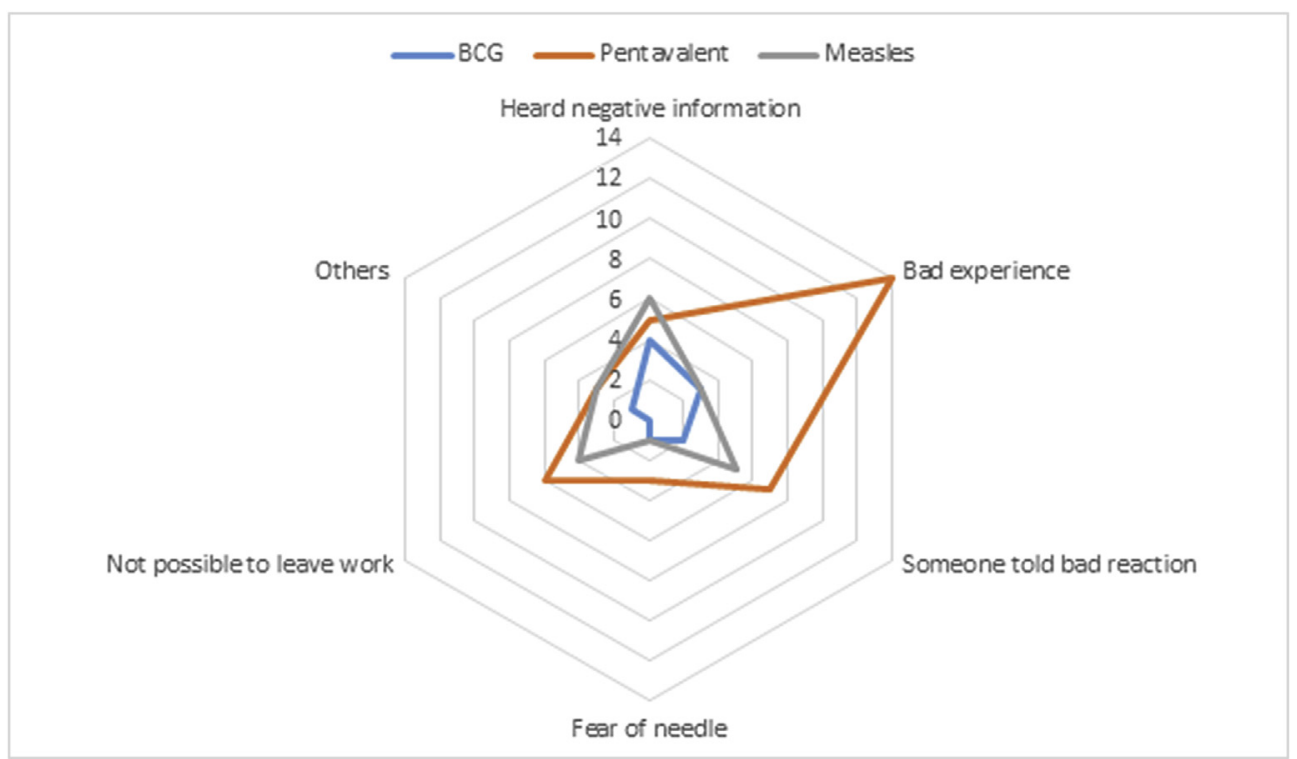

Fig. 1. Radar chart showing prevalence of reasons for vaccine hesitancy or refusal among different vaccines $(\mathrm{n}=69)$. *Others included religious beliefs and lack of trust in vaccine efficacyTotal

number of households were $\mathrm{n}=$ 180.

media and celebrities for mass awareness campaigns. Intensified behavior change communication campaigns through the platforms of community-based events, cultural events, folk dance or local songs reached large scale populations. ${ }^{16}$

Our study revealed that long distances and time are the two prime factors of accessibility linked with the refusal or hesitancy of the vaccine. Congruent to our findings, access or distance to services was mentioned as a reason to under-vaccination in children in low middleincome countries by other studies. ${ }^{17-19}$ While this is remedial with special interventions such as additional vaccine camps in areas with no health facility or increased number of health workers who may visit difficult to reach areas more often. We found that there is a high level of acceptance of vaccines at the level of parents and more than threefourths took their children for vaccination despite hearing negative information about them. This downplays the peer influence as an important factor for vaccine refusal and makes space for quality interaction between health workers and caregivers to increase vaccine uptake. ${ }^{19,20}$ This suggests that a good counselling about vaccine purpose, its adverse events, and immunization schedule can reduce the parental concerns.

It has been stated that a large number of children could not complete their immunization schedules because health services do not regularly reach them. ${ }^{20}$ Similarly, in our study, some ethnic groups reported that health workers do not reach them. This suggests the need for using approaches to reach hidden or marginalized populations with the help of local community-based organizations or by using modern techniques such as geographic information system mapping, etc. This holds importance for socially marginalized populations who have limited access to services including immunization. ${ }^{21}$ We found a high level of support from religious and political leaders for vaccination in our study. In a systematic review based on 202 articles, lack of support from political or religious leaders has been reported to be an important reason for under-vaccination among children. ${ }^{17}$

Among the four basic vaccines, pentavalent and measles were most refused or hesitated vaccines by parents. Previous bad experience with pentavalent vaccine has been highlighted as the most common reason for hesitancy or refusal, which is supported by other studies in the past. ${ }^{22,23}$ Similarly, vaccine hesitancy related to other vaccines such as pertussis, measles, mumps, and rubella (MMR) has been reported in previous studies. ${ }^{24,25}$ Elliott et al. in his study from north India found that measles is the most commonly omitted vaccine in the primary course and first and second boosters of diphtheria, pertussis and tetanus toxoid are most commonly missed vaccines. ${ }^{26}$ In a review published in Lancet measuring vaccine confidence using media surveillance system, it was reported that hesitancy according to vaccine types is countryspecific. ${ }^{27}$ This emphasizes on the need of improving the competency of the frontline health workers in identifying and managing adverse events following immunization (AEFIs). In addition, it calls for effective counselling by frontline workers while giving vaccines to the families about possible adverse events. Government of India has been taking AEFIs very seriously and revised the national guidelines for AEFI surveillance in 2016 and introduced the guidelines for administration of adrenaline in management of severe AEFIs in 2018. ${ }^{28}$

Our findings reinforce the notion that increasing awareness and access to vaccination can help reducing hesitancy. In addition, engaging religious leaders or political leaders to promote vaccination is an important strategy. ${ }^{16}$ Interventions with a comprehensive approach that targets multiple audiences and layers of social interaction are more likely to bring positive results. A recent survey during Intensified Mission Indradhanush in 2017-18 revealed that lack of awareness (45\%), apprehension about adverse events (24\%), vaccine resistance (reluctance to receive the vaccine for reasons other than fear of adverse events) $(11 \%)$ were the common reasons for non-vaccination among under-5 children. ${ }^{5}$

Building upon the proposed dimensions of vaccine hesitancy, namely, awareness, accessibility, and acceptability, we studied their applicability in the rural settings of Odisha. While narrowing down from the wide scale of possible determinants of the vaccination coverage gap, these three dimensions have the possibility of ambiguity and overlap. In a three-year analysis of the data from WHO/UNICEF Joint Report Form (JRF) to determine the reported rate of vaccine hesitancy across the globe, it was found that hesitancy is present in majority of the countries globally. The most common reasons cited were perceived risk-benefit (scientific evidence), lack of knowledge and awareness and religion/culture/gender/socio-economic. This three -year review also suggested that the reasons for hesitancy did not necessarily remain static within a country over time underlining the complexity and variability of hesitancy globally. ${ }^{29}$ 
Strengths and limitations of the study: The study was one of the few community-based studies done in India and especially, among tribaldominated geography of Odisha. Besides, the use of standardized tool and differential vaccine hesitancy make the results novel and acceptable. However, the study had a few limitations. The study included limited questions on awareness dimension, which might not reflect the true scenario. Of the compendium of three different types of survey questionnaires for vaccine hesitancy developed by SAGE working group, we opted core closed questionnaire. However, an open-ended questionnaire was preferred by the group to conceptualize the construct of vaccine hesitancy better. We couldn't use this due to the limitation of skills and interpretation of results in qualitative methodology. We should be cautious in generalizing the results of the study due to the relatively smaller sample size and preferred selection of districts.

\section{Conclusion}

To the best of the knowledge of the author, there are few studies on vaccine hesitancy from India. This research study adds to the existing literature and proposes 3As (awareness, accessibility, and acceptability) as a conceptual framework to identify and classify the reasons for incomplete vaccination coverage. In the current scenario of increasing immunization coverage in India, vaccine hesitancy acts as a missing link in action for the policymakers to achieve cent percent target.

\section{Funding}

Source(s) of support in the form of grants, equipment, drugs, or all of these: The study was a part of implementation science in project JAGRITI supported by Nestlé India (CSR). The donor was not involved in any component of the study.

\section{Declaration of competing interest}

None.

\section{Acknowledgement}

The authors would like to thank the participants of this study for contributing their time. In addition, the authors are grateful for the assistance of field investigators for their help with data collections, and district team lead for his support to field investigators.

\section{References}

1. Rémy V, Zöllner Y, Heckmann U. Vaccination: the cornerstone of an efficient healthcare system. J Mark Access Health Policy. 2015;3:10https://doi.org/10.3402/ jmahp.v3.27041.

2. Larson HJ, Cooper LZ, Eskola J, Katz SL, Ratzan S. Addressing the vaccine confidence gap. Lancet. 2011;378:526-535.

3. Global Alliance for Vaccines and Immunizations (GAVI). GAVI Alliance Progress Report 2010. Geneva, Switzerland: GAVI; 2011.

4. International Institute for Population Sciences (IIPS) and ICF. National Family Health Survey (NFHS-4), 2015-16: India. Mumbai: IIPS; 2017 Available at: http://rchiips. org/NFHS/pdf/NFHS4/India_FactSheet.pdf, Accessed date: 3 January 2019.

5. Gurnani V, Haldar P, Aggarwal MK, et al. Improving vaccination coverage in India: lessons from Intensified Mission Indradhanush, a cross- sectoral systems strengthening strategy. BMJ. 2018;363:k4782https://doi.org/10.1136/bmj.k4782.

6. Hickler B, Guirguis S, Obregon R. Vaccine special issue on vaccine hesitancy. Vaccine.
2015;33:4155-4156.

7. Freed GL, Clark SJ, Butchart AT, Singer DC, Davis MM. Parental vaccine safety concerns in 2009. Pediatrics. 2010;125:654-659.

8. Gowda C, Dempsey AF. The rise (and fall?) of parental vaccine hesitancy. Hum Vaccine. 2013;9(8):1755-1762.

9. National Institute for Transforming India (NITI). Ayog Report. Healthy States and Progressive India: Report on the Ranks of States And Union Territories Delhi: NITI Ayog; 2018 Available at: http://social.niti.gov.in/, Accessed date: 17 March 2019.

10. Panda B, Pati S, Nallala S, et al. How supportive supervision influences immunization session site practices: a quasi-experimental study in Odisha, India. Glob Health Action. 2015;8:25772https://doi.org/10.3402/gha.v8.25772.

11. Government of India. District Census Handbook, Census. 2011; 2011 Available at: http://censusindia.gov.in/2011census/dchb/OdishaA.html , Accessed date: 9 March 2019.

12. World Health Organization (WHO). Immunization Coverage Cluster Survey—Reference Manual. Geneva, Switzerland: WHO; 2005.

13. World Health Organization (WHO). Report of the SAGE Working Group on Vaccine Hesitancy 2014; 2014 Available at: https://www.who.int/immunization/sage/ meetings/2014/october/1_Report_WORKING_GROUP_vaccine_hesitancy_final.pdf , Accessed date: 18 December 2019.

14. Narayanan SS, Jayaraman A, Gopichandran V. Vaccine hesitancy and attitude towards vaccination among parents of children between 1-5 years of age attending a tertiary care hospital in Chennai, India. Indian J Commun. Fam Med. 2018;4(02):31-36

15. Dasgupta P, Bhattacherjee S, Mukherjee A, Dasgupta S. Vaccine hesitancy for childhood vaccinations in slum areas of Siliguri, India. Indian J Public Health. 2018;62(4):253.

16. Jarrett C, Wilson R, Leary MO, Eckersberger E, Larson HJ, SAGE Working Group on Vaccine Hesitancy. Strategies for addressing vaccine hesitancy - a systematic review. Vaccine. 2015;33:4180-4190.

17. Rainey JJ, Watkins M, Ryman TK, Sandhu P, Bo A, Banerjee K. Reasons related to non-vaccination and under-vaccination of children in low and middle income countries: findings from a systematic review of the published literature, 1999-2009. Vaccine. 2011;29(46):8215-8221.

18. Thomson A, Robinson K, Vallée-Tourangeau G. The 5As: a practical taxonomy for the determinants of vaccine uptake. Vaccine. 2016;34:1018-1024.

19. García LDA, Velandia-González M, Trumbo SP, Pedreira MC, Bravo-Alcántara P, Danovaro-Holliday MC. Understanding the main barriers to immunization in Colombia to better tailor communication strategies. BMC Public Health. 2014;14:669https://doi.org/10.1186/1471-2458-14-669.

20. Waisbord S, Larson H. Why Invest in Communication for Immunization: Evidence and Lessons Learned. A Joint Publication of the Health Communication Partnership Based at Johns Hopkins Bloomberg School of Public Health/Center for Communication Programs (Baltimore) and the United Nations Children's Fund. New York. 2005; 2005 Available at: https://www.who.int/immunization/hpv/communicate/why_invest_in communication_for_immunization_unicef_healthcommunicationspartnership_path_ usaid.pdf?ua =1, Accessed date: 15 March 2019.

21. Mukherjee S. Social determinants of child immunization in the states of Rajasthan, Odisha and Madhya Pradesh (India): special focus on social groups. Synopsis of the doctoral dissertation. Available at: https://dspace.gipe.ac.in/xmlui/bitstream/ handle/10973/34589/Sumoni-Synopsis.pdf? sequence = 2\&isAllowed = y , Accessed date: 18 January 2019.

22. Facciolà A, Visalli G, Orlando A, et al. Vaccine hesitancy: an overview on parents opinions about vaccination and possible reasons of vaccine refusal. J Public Health Res. 2019 Mar 11:8(1):1436.

23. Kumar D, Chandra R, Mathur M, Samdariya S, Kapoor N. Vaccine hesitancy: understanding better to address better. Isr J Health Policy Res. 2016;5:2.

24. Atwell JE, Salmon DA. Pertussis resurgence and vaccine uptake: implications for reducing vaccine hesitancy. Pediatrics. 2014;134(3):602-604.

25. Hamilton-West K. Factors influencing MMR vaccination decisions following a mumps outbreak on a university campus. Vaccine. 2006;24:5183-5191.

26. Elliott C, Farmer K. Immunization status of children under 7 years in the vikas nagar area north India. Child Care Health Dev. 2006;32:415-421.

27. Larson HJ, Smith DMD, Paterson P, et al. Measuring vaccine confidence: analysis of data obtained by a media surveillance system used to analyse public concerns about vaccines. Lancet Infect Dis. 2013;13:606-613.

28. Government of India. Revised AEFI guidelines: executive summary. Available at: https://mohfw.gov.in/sites/default/files/Revised\%20AEFI\%20Guidelines \%20Execute\%20Summary.pdf , Accessed date: 16 August 2019.

29. Lane S, MacDonald NE, Marti M, Dumolard L. Vaccine hesitancy around the globe: analysis of three years of WHO/UNICEF Joint Reporting Form data-2015-2017. Vaccine. 2018;36(26):3861-3867. 